\title{
Bifurcation analysis of a model for atherosclerotic plaque evolution
}

\author{
M.A.K. Bulelzai ${ }^{\mathrm{a}}$, J.L.A. Dubbeldamª,*, H.G.E. Meijer ${ }^{\mathrm{b}}$ \\ ${ }^{a}$ Faculty of Electrical Engineering, Mathematics and Computer Science, Delft University of Technology, Mekelweg 4, 2628 CD Delft, \\ The Netherlands \\ ${ }^{b}$ Department of Applied Mathematics, MIRA Institute for Biomedical Engineering and Technical Medicine, University of Twente, \\ Postbus 217, 7500AE, Enschede, The Netherlands
}

\begin{abstract}
We analyze two ordinary differential equation (ODE) models for atherosclerosis. The ODE models describe long time evolution of plaques in arteries. We show how the dynamics of the first atherosclerosis model (model A) can be understood using codimension-two bifurcation analysis. The Low-Density Lipoprotein (LDL) intake parameter $(d)$ is the first control parameter and the second control parameter is either taken to be the conversion rate of macrophages $(b)$ or the wall shear stress $(\sigma)$. Our analysis reveals that in both cases a Bogdanov-Takens (BT) point acts as an organizing center. The bifurcation diagrams are calculated partly analytically and to a large extent numerically using AUTO07 and MATCONT. The bifurcation curves show that the concentration of LDL in the plaque as well as the monocyte and the macrophage concentration exhibit oscillations for a certain range of values of the control parameters. Moreover, we find that there are threshold values for both the cholesterol intake rate $d_{\text {crit }}$ and the conversion rate of the macrophages $b_{\text {crit }}$, which depend on the values of other parameters, above which the plaque volume increases with time. It is found that larger conversion rates of macrophages lower the threshold value of cholesterol intake and vice versa. We further argue that the dynamics for model A can still be discerned in the second model (model B) in which the slow evolution of the radius of the artery is coupled self-consistently to changes in the plaque volume. The very slow evolution of the radius of the artery compared to the other processes makes it possible to use a slow manifold approximation to study the dynamics in this case. We find that in this case the model predicts that the concentrations of the plaque constituents may go through a period of oscillations before the radius of the artery will start to decrease. These oscillations hence act as a precursor for the reduction of the artery radius by plaque growth.
\end{abstract}

Keywords: Atherosclerosis, Bifurcation analysis, Bogdanov-Takens bifurcation, Slow-fast system, Wall shear stress, Oxidized-LDL.

\section{Introduction}

Atherosclerosis is a chronic inflammation of the layers of the artery wall, which gives rise to plaque formation. The plaque is covered with a fibrous cap that may ultimately rupture and lead to a myocardial event. The subject of how atherosclerotic plaques grow and how they may eventually rupture has been investigated for a long time. One mechanism that is responsible for the onset of atherosclerosis is endothelial injury after which subsequent biochemical phenomena take place that trigger the formation of plaques in arteries. The evolution of atherosclerotic plaques is characterized by accumulation of so-called lipid-laden foam cells over time [Crowther 2005] in a part of the arterial wall that is called the intima. The evolution of plaques involves a number of different substances, some of which sare carried with the blood flow and others reside in the layers of the artery. Important constituents include LDL-cholesterol, monocytes, macrophages, cytokines and smooth muscle cells. Besides the biochemical reactions that occur within the plaque mechanical stimuli were

\footnotetext{
${ }^{*}$ Corresponding author

Email address: j.1.a.dubbeldam@tudelft.nl (J.L.A. Dubbeldam )

Preprint submitted to Elsevier
}

March 5, 2014 
shown to play an important role in the development of atherosclerosis [Gijsen et al. 2008, Slager et al. 2005]. The shear stress that is exerted by the blood on the endothelial layer is especially crucial. It was found that high wall shear stress leads to a reduced plaque growth. The growth of a plaque region is therefore anisotropic: the plaque grows predominantly in the downstream direction where the shear stress is much lower than upstream [Ross 1993]. Also during later stages in which smooth muscle cells proliferate and a fibrous cap covering the plaque is constructed, biomechanical factors become important for the stability and elasticity of the cap [Barra et al.]. Mathematical models describing the elasticity of the arterial wall were developed by [Holzapfel et al. 2000].

Although beneficial effects of high wall shear stress on plaque evolution have been demonstrated in experiments [Slager et al. 2005, Gijsen et al. 2008], hardly any mathematical model has been developed that takes biomechanical effects into account. In a recent paper [Bulelzai and Dubbeldam 2012], we put forward an ODE model for the progression of atherosclerosis which includes wall shear stress effects. This model was inspired by a model of [Zohdi et al. 2004], who introduced a phenomenological model to describe plaque evolution by focusing on particle adhesion rather than wall shear stress. Another ODE model was developed by [Ougrinovskaia et al. 2010] for the initiation of the disease. Even though ODE models can never capture all aspects that are relevant for atherosclerosis, ODE models can give qualitative results that can serve as guidelines for clinical experiments. Moreover, ODEs are relevant limiting cases for partial differential equation (PDE) models [Bulelzai et al. 2013]. These PDE-models usually contain parameters whose values are not always known from experiments. Bifurcation analysis can provide clues for the parameter values and the correctness of the model.

In this paper we analyse the dynamics associated with the progression of atherosclerosis by performing a codimension-two bifurcation analysis of the two models proposed in [Bulelzai and Dubbeldam 2012]. These models referred to as model $\mathrm{A}$ and $\mathrm{B}$, respectively, consider the evolution of the plaque in two cases. In model A the dynamics of plaque constituents is modeled without taking into account biomechanical effects. In model B, these effects were included by presuming a wall shear stress dependent recruitment of monocytes. The assumption of a constant throughput of the blood through the artery leads then to a self-consistent model, in the sense that a smaller radius gives rise to larger flow velocity which implies on its turn an increased wall shear stress in a consistent way. The importance of such coupling of the blood flow to the plaque is essential in predicting how the radius of the artery behaves and how the total volume of the plaque evolves.

We organize this paper as follows: In Section 2 we present the models A and B. In Section 3 we focus on the codimension-two bifurcation diagram with two different sets of control parameters. We first consider the dynamics in the case that the LDL-intake rate and the ingestion rate of (oxidized) LDL are the control parameters and later we replace the ingestion rate by the wall shear stress. In both cases, we obtain similar bifurcation diagrams which have a Bogdanov-Takens (BT) point as an organizing center. This allows us to unfold the dynamics for a wide range of parameters. For model B which has a trivial bifurcation diagram, we perform a slow-fast analysis in Section 4 as the radius of the artery evolves on a much longer time scale than the typical time scale associated with the biochemical responses of the plaque constituents. We calculate the slow-manifold and determine the evolution of the artery radius. In Section 5, we discuss the physical interpretation of our bifurcation studies and finally in Section 6 we present the conclusions.

\section{Introduction of models A and B}

\subsection{Model A}

The evolution equations for model $\mathrm{A}$ are given as:

$$
\begin{aligned}
\dot{m} & =\left(\frac{a L}{(1+\sigma)(1+L)}-\epsilon-c\right) m, \\
\dot{M} & =c m-\frac{b M L}{1+L}, \\
\dot{L} & =\frac{d m}{f+m}-e L M-L, \\
\dot{F} & =\frac{b L M}{1+L} .
\end{aligned}
$$


All parameters and variables in the model (2.1) are dimensionless and nonnegative and the dot denotes the time derivative. The physical interpretation of this coupled system of plaque constituents is described in detail in [Bulelzai and Dubbeldam 2012]. Here we will only give a brief explanation of the terms and parameters of the model.

Eq. (2.1a) describes the evolution of the monocytes $(m)$ which enter the arterial wall after a signaling response transmitted by monocytes that are involved in oxidizing LDL molecules [Bulelzai and Dubbeldam 2012]. We assume that the monocytes are converted into macrophages at a dimensionless rate $c$ and may diffuse through the endothial layer into the blood at a rate $\epsilon$. The macrophage concentration $(M)$ is governed by Eq. (2.1b). The macrophages ingest ox-LDL and are next converted into so-called foam cells $(F)$ at a rate $b$. Since macrophages are much larger than monocytes they will typically diffuse much slower and therefore no diffusion term was included in Eq. (2.1b). Finally, the ox-LDL $(L)$ concentration, whose dynamics is governed by Eq. (2.1c), decreases due to the ingestion of ox-LDL by macrophages, at a rate $e$, and increases at a rate $d$ due to the oxidation by monocytes of unoxidized LDL molecules present in the arterial wall. The saturation of this process is taken into account by the parameter $f$. The unoxidized-LDL in the blood penetrates the endothelial layer, which gives rise to a concentration of oxidized-LDL in the arterial wall that we denote by $d$. We assume that the value of $d$ is proportional to the actual intake of cholesterol and therefore we consider $d$ to be a control parameter. Dependence of the dynamics on $b$ and $e$ will also be studied as these parameters influence the 'life time' of the ox-LDL in the plaque, which is a determining factor in the development of atherosclerosis.

We should remark that for the parameter values that we consider $L$ has a rather small value. Therefore we do not include any saturation terms in Eq. (2.1b) as this would introduce an additional parameter whose value is unknown. Furthermore, we notice that the evolution equation for $F$ (2.1d) decouples from the system, so we need only consider the three evolution equations for $m, M$ and $L$. In this model (2.1), which is called model A, in accordance with [Bulelzai and Dubbeldam 2012], the shear stress $\sigma$ is simply considered a parameter. This is in contrast to model B where $\sigma$ is calculated self-consistently using the relation between the radius of the artery and the flow velocity of the blood.

\subsection{Model B}

The previously defined model $\mathrm{A}$ is made self-consistent by requiring that the wall shear stress $\sigma$ is not a control parameter, but rather a dynamic quantity whose evolution is governed by an ordinary differential equation. Assuming a Poiseuille profile of the flow and demanding incompressibility, the wall shear stress $\sigma(t)$ and the artery radius $R(t)$ are seen to be related by

$$
\sigma(t)=\frac{\alpha}{R^{3}(t)}
$$

where $\alpha$ is a constant proportional to the viscosity and the throughput through the artery. For details about the model we refer to [Bulelzai and Dubbeldam 2012].

Model B consists of the evolution equations of $m, M, L$, which are given by Eqs. 2.1(a-c) as in model A, and the evolution of the artery radius, or equivalently, the wall shear stress $\sigma(t)$. The evolution equation for $\sigma$ is derived in [Bulelzai and Dubbeldam 2012] and reads

$$
\dot{\sigma}=-\frac{3}{2} \xi \sigma\left(1-\left(\frac{\sigma}{\alpha}\right)^{\frac{2}{3}}\right)\left[m\left(\frac{a L v_{m}}{(1+\sigma)(1+L)}+c v_{M}-v_{m}(\epsilon+c)\right)+\frac{b M L}{1+L}\left(1-v_{M}\right)+v_{L}\left(\frac{d m}{f+m}-e M-L\right)\right] .
$$

In Eq. (2.3) $\xi$ is a small parameter and therefore model B constitutes a so-called slow-fast system with $m, M, L$ the fast variables and $\sigma$ the slow variable. We further remark that the parameters $v_{i}$ denote the volumes of the different constituents with respect to the foam cells and therefore $v_{i} \ll 1$. Hence a good approximation of Eq. (2.3) is

$$
\dot{\sigma}=-\frac{3}{2} \xi \sigma\left(1-\left(\frac{\sigma}{\alpha}\right)^{\frac{2}{3}}\right) \frac{b M L}{1+L}
$$


We can summarize model B by the following four evolution equations:

$$
\begin{aligned}
\dot{m} & =\left(\frac{a L}{(1+\sigma(t))(1+L)}-\epsilon-c\right) m, \\
\dot{M} & =c m-\frac{b M L}{1+L} \\
\dot{L} & =\frac{d m}{f+m}-e L M-L, \\
\dot{\sigma} & =-\frac{3}{2} \xi \sigma\left(1-\left(\frac{\sigma}{\alpha}\right)^{\frac{2}{3}}\right) \frac{b M L}{1+L} .
\end{aligned}
$$

\section{Bifurcation analysis}

In this section we will study the dynamics of model A, by first constructing a codimension-two bifurcation diagram for model $\mathrm{A}$. The bifurcation diagram obtained for model A will serve as a starting point for our slow-fast analysis of model B. We proceed to discuss the general features of the dynamics and the bifurcation diagram in the next subsection. Details are provided in the Appendix.

\subsection{Codimension-two bifurcation diagram of model $A$}
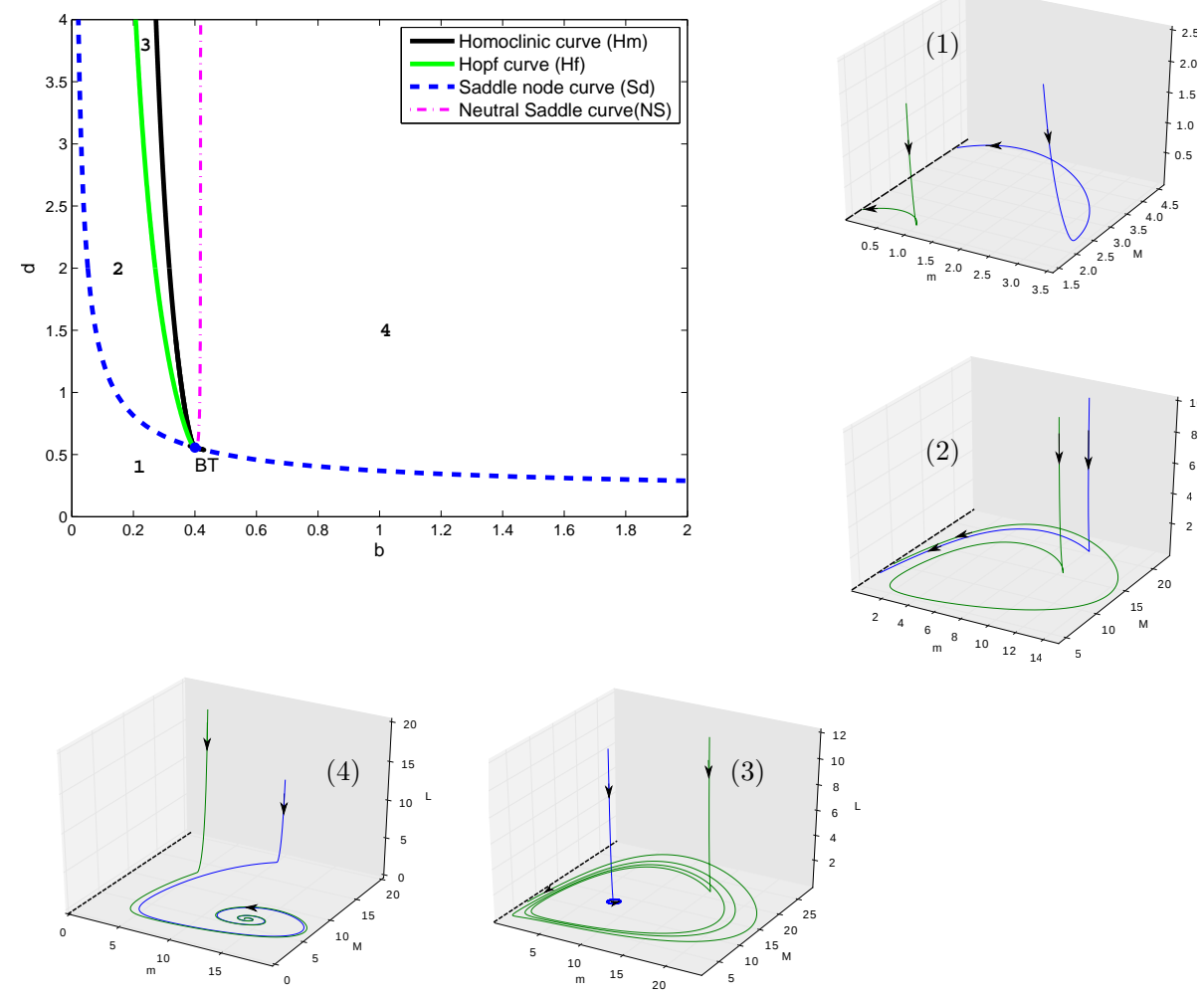

Figure 1: The two-dimensional bifurcation diagram for $e=1$ (and $a=f=1, \epsilon=0.01$ and $c=0.05, \sigma=1$ ). A Bogdanov-Takens point $(B T)$ acts as an organizing center. The Hopf curve $(H)$, the homoclinic curve $(H o m)$ and the saddle node curve $(S)$ intersect at $B T$. Simulations corresponding to regions (1-4) are also shown. The line of marginal equilibria $l$ is shown with dashed black lines in the portraits and colors green and blue, show the two orbits starting from different initial conditions. The normal form coefficient for BT point (see Appendix), $s=\operatorname{sign}\left(a_{1} / b_{1}\right)$, is positive here; the calculations of the nomrmal form coefficients are relegated to appendix A. 
The system of differential equations given by Eqs. (2.1) possesses infinitely many equilibria, which are separated into two classes. The first class of equilibria (infinitely many), which we denote by type $I$, are of the form $(m, M, L)=\left(0, M_{0}, 0\right)$, where $M_{0}$ can have any non-negative value; we will denote this line of solutions with $l$ in this paper. The second class of equilibria (type $I I$ ) corresponds to two points with $m \neq 0$, which we will denote by $\left(m^{ \pm}, M^{ \pm}, L^{*}\right)$.

We study the existence and stability of the equilibria by varying two parameters. First, we consider the bifurcation diagram in the $(b, d)$-plane treating both the ingestion of oxidized cholesterol by macrophages and the intake rate of LDL particles as control parameters. The reason for choosing $d$ is that it is the natural parameter of the model as it determines the total flux of LDL cholesterol entering into the plaque region and $b$ because it determines the effect of macrophage secretory products such as, Interleukin- 6 which determines how fast LDL-particles are eliminated from the plaque region [Frisdal et al. 2011].

Moreover, the two-dimensional bifurcation diagram allows a Bogdanov-Takens (BT) point to be identified which acts as an organizing center. As general references to the bifurcations we encounter see for example [Guckenheimer and Holmes 1983, Kuznetsov 1998].

There is a critical value of $e$ such that the Bogdanov-Takens bifurcation is degenerate, i.e. there is a codimension-three bifurcation. Hence, we find qualitatively different bifurcation diagrams depending on the value of $e$. We therefore discuss both cases separately. The parameters $a, f, \epsilon, c$ are kept constant throughout the paper and their values are set to $a=f=1, \epsilon=0.01$ and $c=0.05$, which were also used in [Bulelzai and Dubbeldam 2012]. As the values of $f$ and $a$ are not known from experiments their values were set to unity in order to obtain the correct timescale for the progression of atherosclerosis; we also checked that the bifurcation diagrams we obtained were robust against variations in the values of $a$ and $f$. For the $(b, d)$-diagram, the parameter $\sigma$ is fixed and set to 1.0 ; for the $(\sigma, d)$-diagram, the value of the parameter $b$ is fixed and set to 0.7 in this paper.

\subsubsection{Bifurcation diagram for $e=1$}

For the values of the parameters mentioned above, we find the two-dimensional bifurcation diagram displayed in Fig. 1, using the numerical continuation package AUTO07 [Doedel and Oldeman 2012]. The phase portraits that correspond to regions (1-4) in Fig. 1, are depicted at the borders of Fig. 1.

The dynamics in the respective regions (1-4) are as follows. In region (1) there are no equilibria beside the ones on the invariant manifold $l$, so all trajectories will end there, independent of the initial condition. For the atherosclerosis this implies that in this regime any perturbation in $m, M$, will lead to a small increment in the total plaque volume, which is reflected by a small increase in the foam concentration $F$ (Equation 2.1d), which acts as a reservoir for the plaque components.

When $d$ is increased so that the curve $S$ is crossed, a saddle node bifurcation occurs in which two new equilibria are generated, both of which are repelling. This implies that in region (2) the phase portrait is very similar to that of region (1). Only when $d$ is further increased, and hence the Hopf-curve $(H)$ is crossed and region (3) is entered, the dynamics are very different. One of the equilibria born in the saddle node bifurcation turns stable and an unstable limit cycle emerges through a subcritical Hopf bifurcation. The unstable limit cycle separates the basin of attraction of the invariant line $l$ and the attracting equilibrium $\left(m^{+}, M^{+}, L^{*}\right)$, and therefore the system is bistable. An even further increase in $d$ leads to a homoclinic bifurcation Hom in which the stable and unstable manifolds of the equilibrium $\left(m^{-}, M^{-}, L^{*}\right)$, connect after which the unstable limit cycle vanishes. When we enter region (4), we are left with an attracting focus and a saddle besides the invariant line $l$. In this region (4) bistability therefore remains, but the basin of attraction of the attracting equilibrium $\left(m^{+}, M^{+}, L^{*}\right)$ has expanded as can be seen from the phase portrait.

\subsubsection{Bifurcation diagram for $e=5$}

When we change the value of the parameter $e=1$ to $e=5$, we have a diagram in which the positions of $H o m$ and $H$ curve are interchanged. In this case, it is known that there will be a point $G H$ on the Hopf curve $(H)$ where the first Lyapunov coefficient vanishes [Dumortier et al. 1991]. At this point the Hopf bifurcation changes from subcritical to supercritical. Moreover, there will be a curve (limit point of cycles curve) which connects the point $G H$ to $N S$, the point where the neutral saddle curve and the homoclinic curve meet. This gives rise to four new regions (5) and (6), (7) and (8) where regions (6), (7) and (8) are so close to the 
homoclinic and Hopf curves that they cannot be discerned in Fig. 2(a), but in the qualitative sketch 2(b) their size has been exaggerated to clarify their presence.

The phase portraits corresponding to region (1-8) are shown in Fig.3 both quantitatively and qualitatively. We remark that we can draw two-dimensional phase portraits as the saddle point has a two-dimensional stable manifold for the parameter values that we consider. In the sketch in Fig. 3 the vertical direction corresponds to $L$ and the horizontal axis to the $m$-direction. The line $l$ of equilibria is denoted by a solid filled circle, the saddle point by an open square and stable (unstable) focus by a filled (open) circle. Stable limit cycles are designated by solid closed curves and unstable ones by dashed curves. Regions (1-4), which were also
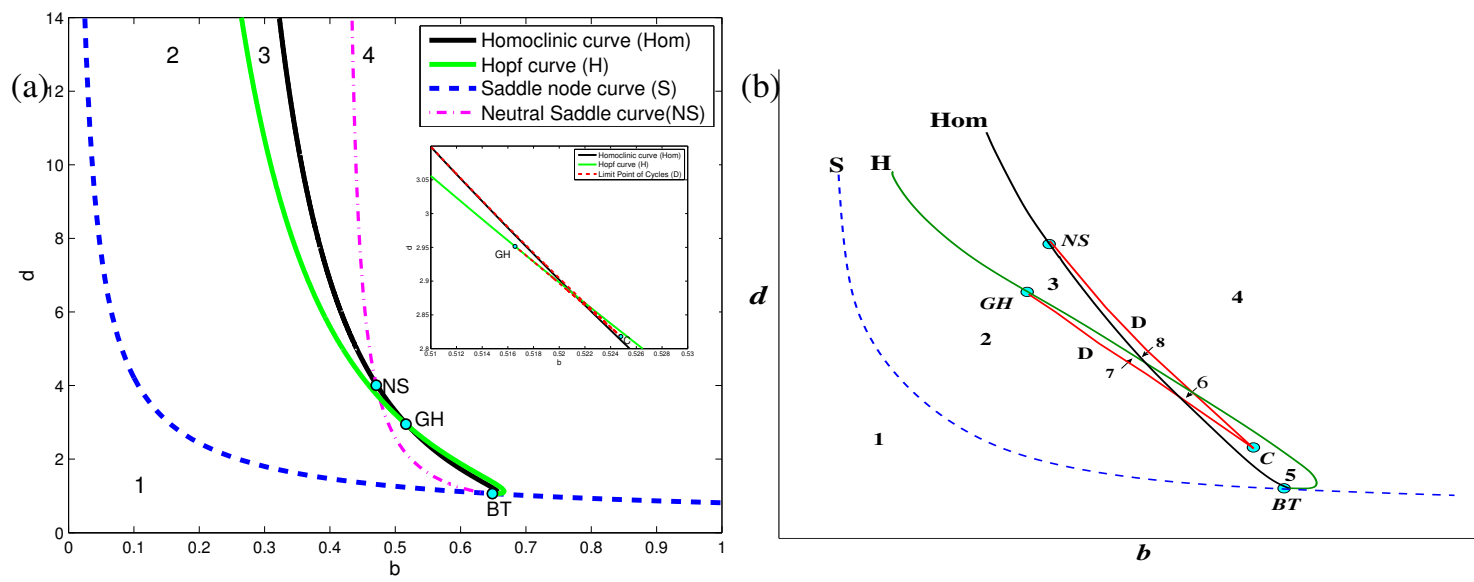

Figure 2: Two-dimensional bifurcation diagram for $e=5$, (and $a=f=1, \epsilon=0.01$ and $c=0.05, \sigma=1$ ) (a) and a qualitative sketch of the region of the bifurcation diagram near where the curves intersect (b). A Bogdanov-Takens point $(B T)$ acts as an organizing center. The Hopf curve $(H)$, the homoclinic curve $(H o m)$ and the saddle node curve $(S)$ intersect at $B T$. The inset and (b) show small parameter regions (5)-(8) that are bounded by a curve of limit point of cycles bifurcations $(D)$, which originates in a cusp point $(C)$, connecting the degenerate Hopf point $(G H)$ with the neutral saddle point $(N S)$. The sign of the critical normal form coefficients for the BT point, $s=\operatorname{sign}\left(a_{1} / b_{1}\right)$ is negative in this case (See Appendix A).

present for the case $e=1$, are accessed when $d$ is sufficiently large. This implies that for sufficiently large cholesterol intake no qualitative difference between the $e=1$ and $e=5$ scenario is observed. This can be understood by realizing that $e$ determines how fast the LDL disappears in the presence of macrophages. A larger cholesterol intake counteracts an enhanced value of $e$ and therefore the phase portraits (1)-(4) are still present for $d$ large enough. If we cross the curve $D$ of limit point of cycles bifurcations by going from region (2) to region (7), we see from the two-dimenional sketches in Fig. 3 that two limit cycles are created: a stable and an unstable one. The equilibria remain of saddle and unstable focus type. There is bistability as there is one stable limit cycle as well as the line of equilibria $l$. We remark that in Fig. 3 we can distinguish a stable limit cycle near the unstable focus (diamond) and the orbit starting near the saddle that eventually arrives in l. When crossing the homoclinic curve (Hom) from region (7) one enters region (6), where two stable limit cycles are present and the two type II equilibria remain of the same type as in (6); the system again exhibits multistability. Region (5) can be accessed by crossing the curve $D$ of limit point of cycles bifurcations in which a stable and an unstable limit cycle collide, leaving the system with a single stable limit cycle and the stability of equilibria unaltered.

The dynamics in the remaining region (8) can be explored if one crosses the curve $D$ of limit point of cycles bifurcations from region (4). In this case the stability of the equilibria remain unaltered and a pair of limit cycles, one stable and one unstable, is created. Again multistability is found. This time between the stable focus, equilibria of class I and the stable limit cycle. In panel 8 of Fig. 3 the stable equilibrium and limit cycle are shown. We should remark that regions (5), and especially (6), (7) and (8) cover a very small part of parameter space, which suggests that these regions will probably not be accessible to experiments and that most clinical results will pertain to regions (1-4). 


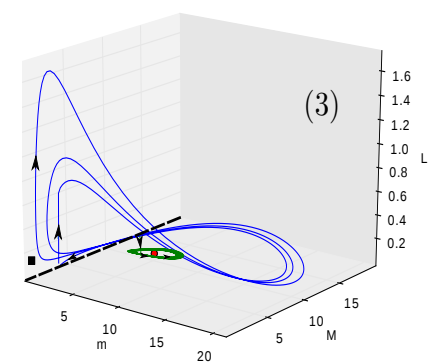

$(4)$
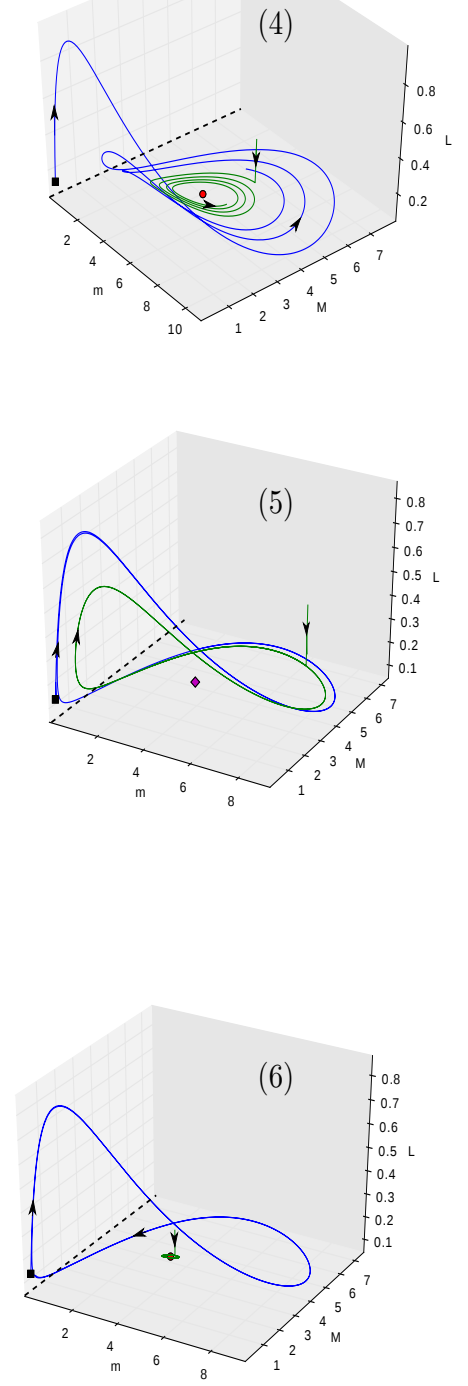
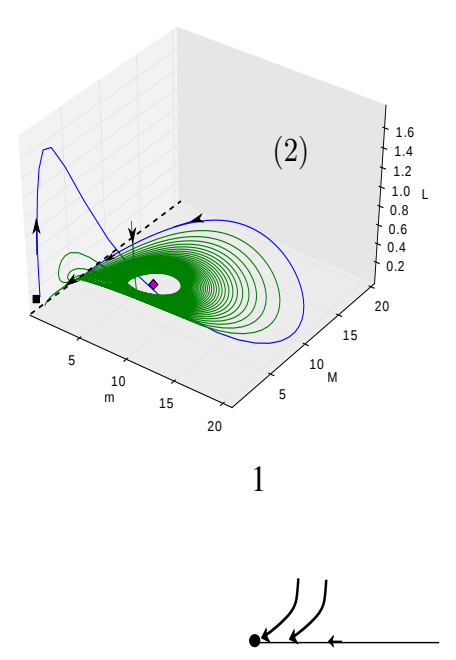

3

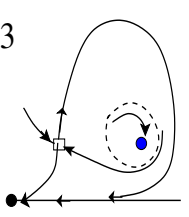

5

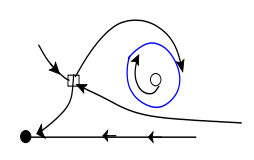

7

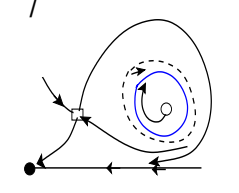

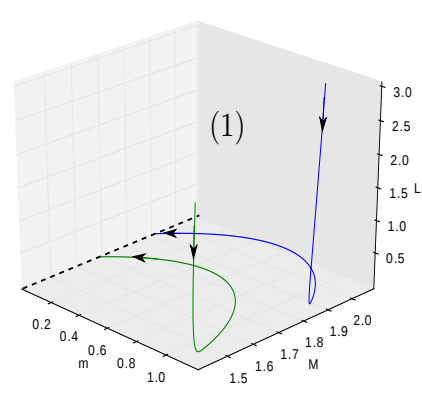
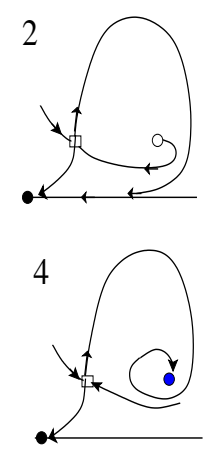

6

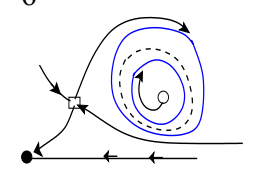

8

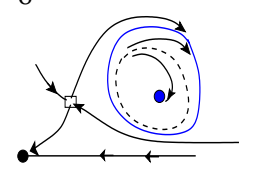

Figure 3: Both three-dimensional and sketches of the two-dimensional phase portraits for 8 different parameter regions in model A. Green and blue lines stand for two different initial conditions that were used to obtain the 3D-portraits. The line of marginal equilibria $l$ is shown with dashed black lines in the portraits, the diamond denotes an unstable focus and the filled circle a stable focus; the colors green and blue, show the two orbits at different initial conditions. In the sketch the line $l$ of equilibria is denoted by a solid filled circle, the saddle point by an open square and stable (unstable) focus by a filled (open) circle. Stable limit cycles are designated by solid closed curves and unstable ones by dashed curves. 


\subsection{Bifurcation diagram in the $(\sigma, d)$-plane}

Instead of considering the bifurcations of system (2.1) in the $(b, d)$-plane, we can also study the bifurcations in the $(\sigma, d)$-plane, where $\sigma$ determines the wall shear stress which is an important quantity as it determines the biomechanical effects. Moreover, the parameter $d$ sets the timescale in the model and $\sigma$ is slowly varying in the self-consistent model B. The diagram obtained for this set of parameters will therefore also be useful in analyzing the dynamics of model B, which is the subject of the next section. We first briefly discuss Fig. 4 which shows the bifurcation curves for varying $\sigma$ and $d$. As can be seen by comparing Fig. 4(a) and (b) with Figs. 1 and 2 the diagrams are very similar. The labels (1)-(4) correspond to the portraits that were shown in Fig. 1. The marginal fixed point $\left(0, M_{0}, 0\right)$ exists for all parameter values and is a global attractor below the saddle node-curve $(S)$. Above the $S$-curve there are the two nontrivial fixed points of type $I I$. One is a saddle whose stable manifold is a separatrix for the basin of attraction between the marginal fixed point and another steady state. This second steady state is stable for sufficiently high values of $\sigma$. For decreasing values of $\sigma$ we encounter a subcritical Hopf bifurcation and the only attractor is the invariant line $l$ of marginally stable equilibria. The Hopf curve meets the $S$-curve at a Bogdanov-Takens bifurcation from where also a homoclinic bifurcation curve emerges. There exists an unstable periodic solution for parameter values between the Hopf and the homoclinic curve. Note that the Hopf curve crosses the $d$-axis at $d \approx 0.8$. This intersection point moves to very high values of $d$ when decreasing $b$. Increasing $b$, on the other hand, shifts the Hopf curve and BT-point to negative values of $\sigma$. Next we set $e=2$ and keep $d$ and $\sigma$ as bifurcation parameters. The main qualitative change in the bifurcation diagram is that the Hopf curve close to the BT-point is now supercritical. This is accompanied by the appearance of a generalized Hopf (GH) and a neutral saddle (NS) point on the Hopf and homoclinic curves, respectively. These codimension-two bifurcation points are connected by a short curve corresponding to a curve of limit point of cycles $(D)$ bifurcations. This implies that there is a region with stable oscillations limited by the $\operatorname{Hopf}(H)$, homoclinic $(H o m)$ and limit point of cycles $(D)$ curve. We note that for higher values of $e$ the curve $D$ exhibits a cusp bifurcation.
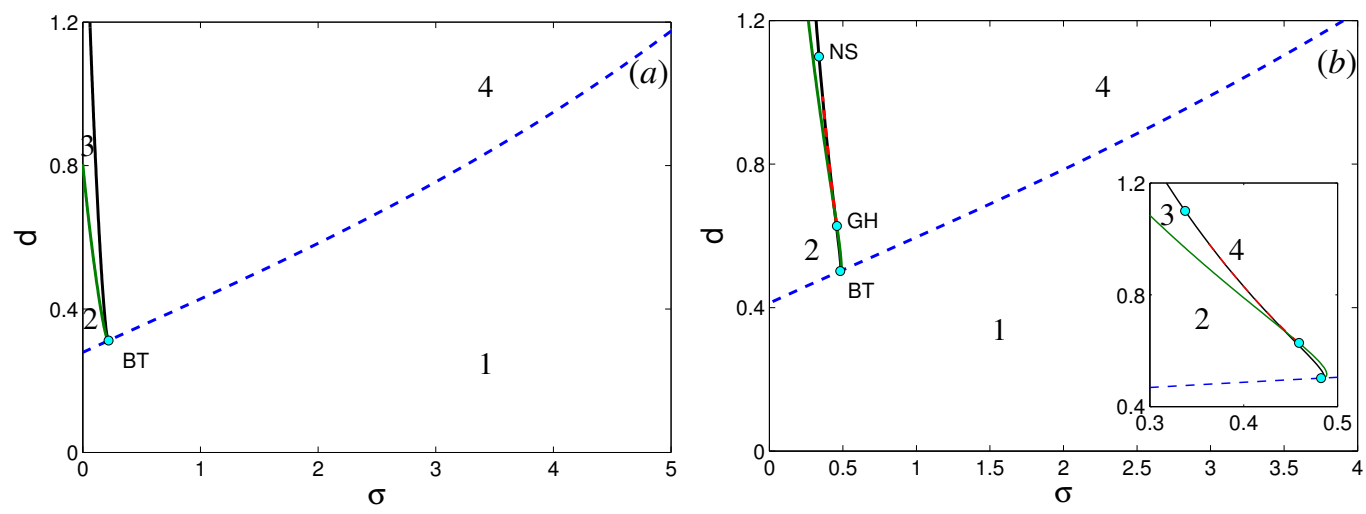

Figure 4: Bifurcation diagrams for $e=1$ (a) and $e=2$ (b) The other parameter values are $a=f=1, c=0.05, \epsilon=0.01$ and $b=0.7$. The significance of the curves is as follows: saddle node curve (dashed blue), Hopf curve (solid green), homoclinic curve (solid black) and the curve of limit point of cycles (dashed red). Codimension-two bifurcation points are Bogdanov-Takens (BT), Generalized Hopf (GH), neutral homoclinic loop (NS) indicated by light blue markers. The sign for second Lyapunov coefficient for GH point is negative. The sign for critical normal form coefficients, $s=\operatorname{sign}\left(a_{1} / b_{1}\right)$ is positive for $e=1$ and negative for $e=2$; see also Appendix A.

\section{Slow-fast analysis of model B}

The system (2.5) constitutes a slow-fast system with a small parameter $\xi$. Slow-fast systems have attracted much interest lately. The complicated geometry of slow manifolds near a folded node was numericaly studied in [Desroches et al. 2008]. The applications of slow-fast dynamics, and the immediately related rampedbifurcation theory, to climate models has recently lead to the surprising prediction of a so-called compost bomb instability by [Wieczorek et al. 2011]. We apply a similar analysis here. The equations (2.5)(a-c) constitute the fast system. The slow system (d) is analyzed by scaling time $\tau=\xi$, where $\tau$ is the slow time scale. The new system of differential algebraic equations can be summarized as

$$
\xi \dot{x}=f(x, \sigma), \quad \dot{\sigma}=g(x, \sigma),
$$


where $x=(m, M, L)$ are the fast variables and $f(x, \sigma)$ and $g(x, \sigma)$ are smooth and continuously differentiable functions given by the system (2.5). According to Fenichel's first theorem, the reduced slow system in the limit of $\xi \rightarrow 0$ is given as:

$$
0=f(x, \sigma), \quad \dot{\sigma}=g(x, \sigma) .
$$
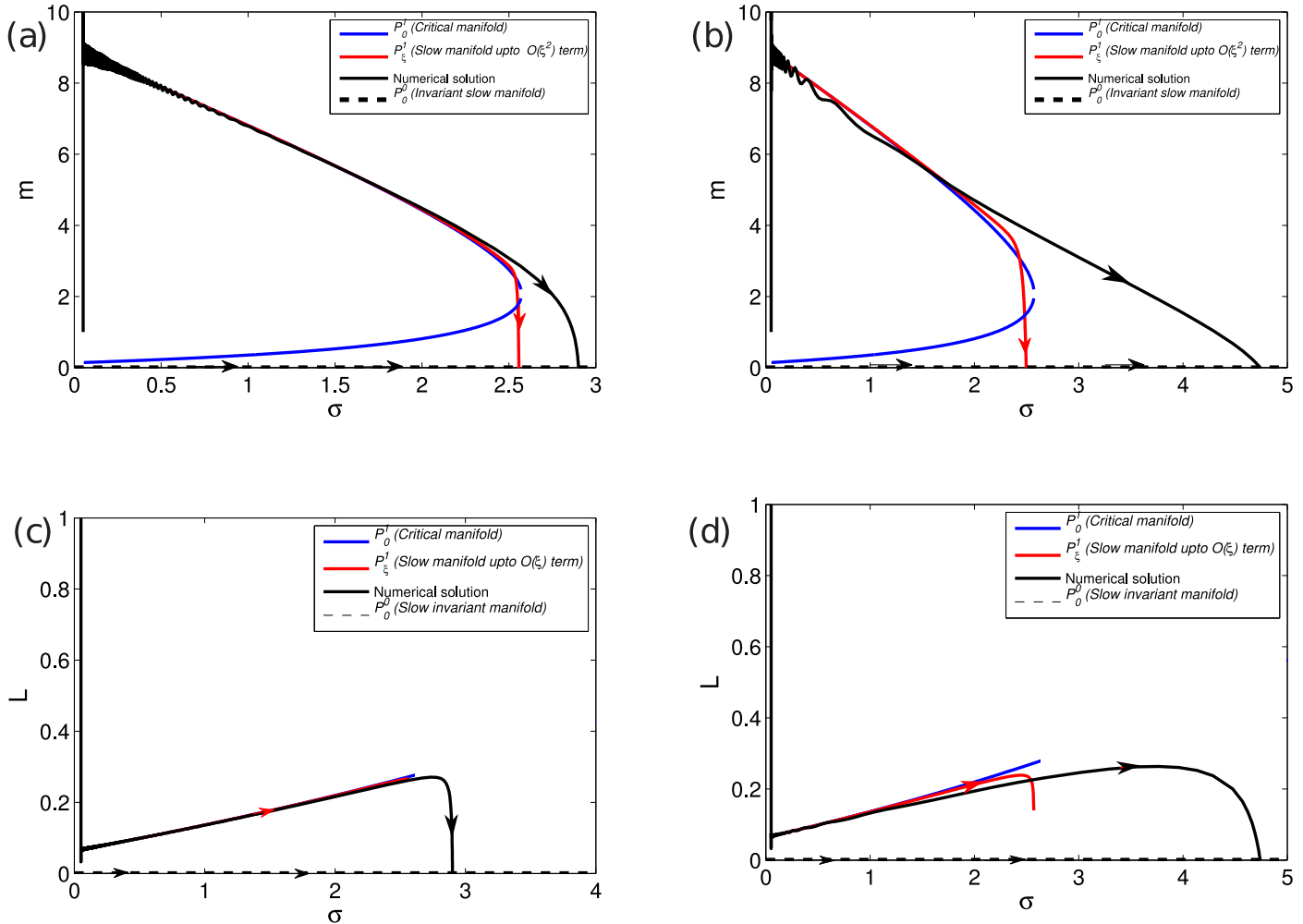

Figure 5: (a,c) with $\xi=0.0002$ and (b,d) with $\xi=0.002$; Slow manifold calculated for two different values of $\xi$ using fixed values for parameters, which are already mentioned in this paper. The numerical solution (black), approximation of slow manifold (red) are shown along with the critical manifold (blue). The line $m=0$ and $L=0$ (dashed) are also shown, which constitute the invariant slow manifold.

The critical manifolds are obtained by solving the equations $f(x, \sigma)=0$ and read

$$
\begin{gathered}
P_{0}^{0}=\left\{(m, M, L) \mid m=0, M=M_{0}, L=0\right\} \\
\text { and } P_{0}^{l}=\left\{(m, M, L) \mid m=m_{0}^{+}(\sigma), M=M_{0}^{+}(\sigma), L=L_{0}^{*}(\sigma)\right\} .
\end{gathered}
$$

The time evolution of the reduced (one-dimensional) system, is obtained by evaluating $g(x, \sigma)$ on the slow manifold $P_{0}^{1}$, which yields

$$
\dot{\sigma}=-\frac{3 b M_{0}^{+}(\sigma) L_{0}^{*}(\sigma) \sigma}{2}\left(1-\left(\frac{\sigma}{\alpha}\right)^{\frac{2}{3}}\right)
$$

The slow manifolds $P_{\xi}^{(0,1)}$ exist by the virtue of geometric singular perturbation theory, e.g. see [Arnold and Jones 1994]. The superscripts in the manifold $P_{\xi}^{(0,1)}$ stand for the two different classes of equilibria that we mentioned earlier. For the type I equilibria, which do not depend on $\xi$, this manifold $P_{\xi}^{(0,1)}=P_{0}^{0}$ (dashed black line in Fig. 5). For the nontrivial equilibria we find the first and second order perturbations in $\xi$ by the substitution $P_{\xi}^{l}(\sigma)=P_{0}^{l}(\sigma)+\xi P_{l}^{l}(\sigma)+\xi^{2} P_{2}^{l}(\sigma)$ ([Kaper et al 2002, Gear et al 2005, Wieczorek et al. 2011, Guckenheimer et al. 2012]) and find:

$$
\begin{aligned}
O(\xi): & \left(D_{x} f\right) P_{l}^{l}=\left(D P_{0}^{1}\right) g \\
O\left(\xi^{2}\right): & \left(D_{x} f\right) P_{2}^{l}=\left(D P_{l}^{l}\right) g+\left(D P_{0}^{l}\right)\left(\left(D_{x} g\right) P_{l}^{l}\right)-\frac{1}{2}\left(D_{x}^{2} f\right)\left(P_{l}^{l}, P_{l}^{l}\right)
\end{aligned}
$$


where $D_{x} f$ is $3 \times 3$ matrix of partial derivatives $\partial f_{i} / \partial x_{j},\left(D P_{0}^{1}\right)$ is $3 \times 1$ matrix of partial derivatives $\partial P_{0}^{1} / \partial x_{i}$, $\left(D_{x} g\right)$ is $1 \times 3$ matrix of partial derivatives $\partial g / \partial x_{i}$. When we solve Eqs. (4.4) we obtain:

$$
\begin{gathered}
P_{l}^{l}=\left\{(m, M, L) \mid m=m_{1}^{+}(\sigma), M=M_{1}^{+}(\sigma), L=L_{1}^{*}(\sigma)\right\} \text { and } \\
P_{2}^{l}=\left\{(m, M, L) \mid m=m_{2}^{+}(\sigma), M=M_{2}^{+}(\sigma), L=L_{2}^{*}(\sigma)\right\},
\end{gathered}
$$

where $M_{i}^{+}(\sigma), m_{i}^{+}(\sigma)$ denote the $i$-th order approximation of the stable equilibrium. For details of the calculations we refer the reader to Appendix B.

\subsection{Dynamics with shear stress}
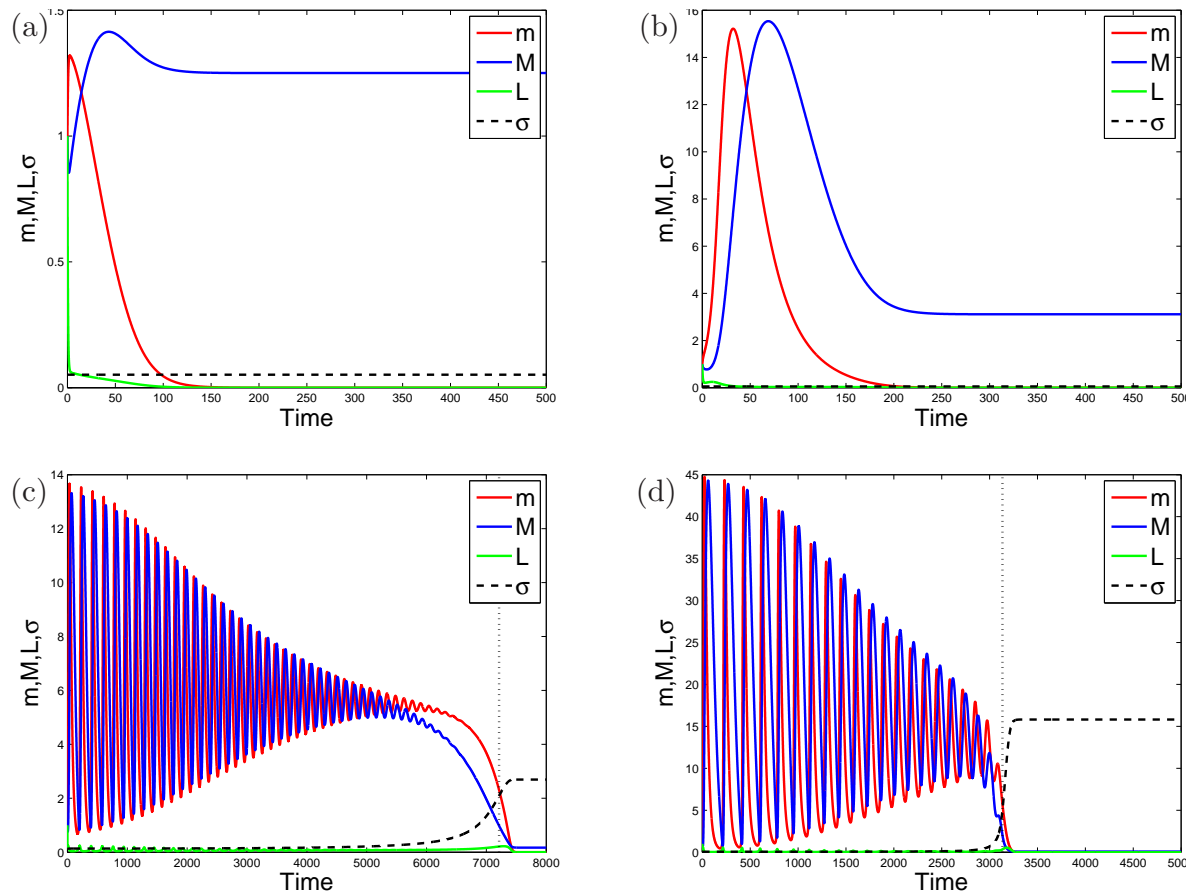

Figure 6: Time-evolution $\sigma$, the monocytes, ox-LDL macrophage and concentrations for (a) $d=0.2$; (b,c) $d=0.6$; (d) $d=1.2$. We choose $\xi=0.002$ and $\alpha=0.05$ and all other parameters are mentioned in the text except $\alpha=0.13$ in (c). The vertical dashed line indicates that $\sigma$ crossed the critical value. Note the different time-scales of the various simulations.

In the full system we show several characteristic simulations starting with $(m, M, L, R)=(1,1,1,0.99)$ for various values of $d$. These initial values are chosen such that the orbit does not always jump immediately to the marginal fixed point. The first case is with low $d$. For any positive $\sigma$ there is no nontrivial steady state and the orbit quickly returns to the marginal fixed point, see Fig. 6(a). The net change of $R$ is small and the artery stays open. The second case is for medium values of $d$, i.e. above the curve $S$, but below the Hopf curve $H$. Then it depends on the choice for $\alpha$ which scenario occurs as it determines the initial $\sigma$ value. For $d=0.6$ and $\alpha=0.05$ the nontrivial steady states are both unstable and the trajectory shows a transient outburst of monocytes but then the orbit approaches the marginal fixed point, see Fig. 6(b). Here too, the artery radius remains large. Changing $\alpha=0.13$, we observe oscillations whose amplitude slowly diminishes until $\sigma$ is too large and nontrivial dynamics is no longer supported, see Fig. 6(c). For higher values of $d$, there is a stable nontrivial steady state. The orbit displays damped oscillations while converging to it. Then $R$ decreases and $\sigma$ increases so that the steady state moves and the orbit tries to follow it until $\sigma$ comes close to a critical value determined by the $(S)$ curve, see Fig. 6(d). In the latter two cases the final radius $R$ is considerably smaller. In Fig. 7, we have presented two graphs obtained with two different initial conditions with $e=2$. In Fig. 7(a), the initial value for radius is 0.99 and in Fig. 7(b) we set $R(0)=0.999$ and changed the value of $\alpha$ accordingly. 

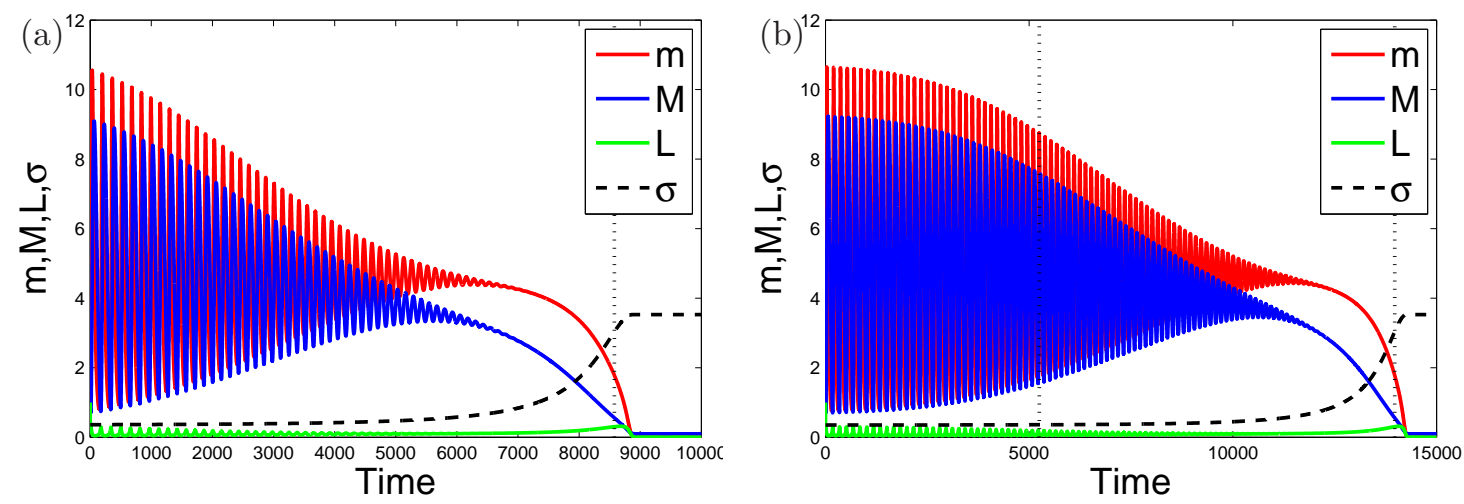

Figure 7: $(a)$ with $R(0)=0.99,(b)$ with $R(0)=0.999$; Time-evolution of shear stress, monocytes, ox-LDL and macrophages concentrations for $d=1.0, e=2, \alpha=0.35$ and with two different initial conditions. The vertical dashed line indicates that $\sigma(t)$ crosses a critical values. In (b), since $\sigma$ is initially very low, first the Hopf curve is crossed (shown dotted) while in $(a)$, due to a larger initial perturbation, only crossing of the $S$-curve can be detected.

\section{Physical interpretation}

In this section we discuss the physical interpretation of the phase portraits in regions (1-4) and bifurcation diagrams. The small regions are not discussed as they will be very hard to find experimentally. In both models $\mathrm{A}$ and $\mathrm{B}$, a high value of cholesterol intake $(d)$ increases the oxidized-LDL particle concentration, which in turn increases the volume of the plaque. The two parametric bifurcation diagrams in this paper, shed more light on the ingestion rate of macrophages and effects of endothelial shear stress. We employed the functional form of $\frac{1}{\left(1+\sigma_{w} / \sigma_{0}\right)}$ in the evolution of the monocytes to model the effect that low shear stress at the endothelium causes more plaque accumulation. It is well established that low or oscillatory wall shear stress is a plaque promoting condition [Gijsen et al. 2008, Slager et al. 2005]. Regions of high shear stresses do not experience plaque growth while regions of low and oscillatory wall shear stress do.

We first comment on the bifurcation diagrams corresponding to model $\mathrm{A}$ and next discuss the consequences for model B. It is evident from Figs. 1, 2 and 4 that region 1 is the healthy region in model A. The critical value of $d$ increases (following the saddle-node curve) with wall shear stress (see Fig. 4) and decreases with $b$, whose value determines how fast macrophages are transformed into foam cells. The dependence of the critical value of $d$ on $\sigma$ is as expected, as high shear stress is in general favorable to diminish plaque growth and the dependence of $d_{c r i t}$ on $b$ is also no surprise as faster ingestion of LDL by macrophages will result in enhanced plaque formation. The marginally attracting manifold $l$ corresponds to both monocytes and ox-LDL concentrations zero. Perturbing the system in this state will only alter the macrophage concentration to a new constant value.

In region 2 model $\mathrm{A}$ has an unstable node. In the $(b, d)$ diagram this region corresponds to conversion rates of macrophages that are sufficiently large to deplete the cholesterol in the plaque and therefore the plaque will only experience growth during a small period of time, after which the plaque turns stationary again. In the $(\sigma, d)$-diagram, region 2 correponds to wall shear stress values that are sufficiently low not to have any other stable states except the invariant line $l$. This region marks a parameter range in which plaques do not grow indefinitely, but behave similarly to region 1 in which a perturbation in LDL can lead to a limited decrease of the artery radius.

In region 3 model A has an unstable limit cycle and a stable focus. The presence of a stable focus indicates that the plaque may start to grow indefinitely, depending on the initial conditions. If the intial values of the monocytes, macrophages and LDL are such that they are in the region of attraction of the focus, the plaque will grow indefinitely with oscillations in the concentrations of the plaque constituents that gradually die out. When the initial conditions are outside the attraction zone of the focus, a limited plaque growth is established, which is in general preceeded by transient oscillations in the concentration of plaque constituents. The same reasoning is true for region 3 in the $(\sigma, d)$-plane, which is surprising since a large $\sigma$ value is considered to lead to less plaque growth. In our model, $\sigma$ is also responsible for the recruitment of monocytes from the 
blood in the plaque. Increased values of $\sigma$ imply an impeded recruitment of monocytes and hence only a moderate population of macrophages. These macrophages ingest LDL, but due to the small value of $b=0.7$, the LDL survives long enough to enhance the LDL concentration, which on its turn leads to an increased monocyte concentration. The oscillations that are created will in general die out to zero, but for particular initial values they can approach a constant nonzero value and the plaque will grow indefinitely. We should stress here that the bifurcation diagram Fig. 4 was made for $b=0.7$. When $b>1$ the BT-point will disappear and the dependence of the dynamics on $\sigma$ is in accordance with expectations. This would suggest that for real arteries $b>1$.

If the system is in region 4, the plaque will in general grow indefinitely, although for certain initial conditions the plaque growth ends in finite time. Region 4 can be accessed for high values of $\sigma$ or high values of the ingestion rate $b$. The fact that large values $b$ are detrimental can easily be comprehended. The larger the value of $b$ the more macrophages are converted into foam cells and consequently a large plaque growth results. The dependence on $\sigma$ is explained similarly to the behavior in region 3. In region 4 the wall shear stress is large enough to sustain a certain amount of monocytes that induces a small population of macrophages. Therefore the LDL particles can survive long enough to attract more monocytes and LDL molecules into the plaque, which leads to a growing plaque volume for $b=0.7$. Again we remark that this dependence on $\sigma$ disappears for $b>1$.

Larger perturbations may push the dynamics into the bassin of attraction of the nontrivial state $\left(m^{+}, M^{+}, L^{\star}\right)$. In model B all solutions will finally reach the invariant manifold $l$. So we discuss the transients that arise when the system is perturbed from its initial state, that we take to be on $l$. Small perturbations to the system in this state will again die out, but the artery radius will decrease a little. Nevertheless, several consecutive perturbations may lead to a sigificant reduction of artery radius.

For model B the dynamics in region 2 stabilizes after transient oscillations in the constituents of the plaque. The wall shear stress first increases as depicted in Fig. 6(b), and then stabilizes to a constant value. This means that here the radius of the artery has decreased and subsequent perturbations in the plaque constituents may cause further reductions in the artery size. For short times, the unstable limit cycle implies a continuous periodic growth of plaque constituents, but eventualy the radius reduction of the artery, or equivalently, the growth of $\sigma$, will come to a halt, when $\sigma(t)$ crosses the $S$-curve. Starting from region 3 or 4 in model B corresponds to assuming higher values of $\alpha$ and hence a higher flux through the artery. Depending on the value of $b$ we find that the radius reduction increases with $\alpha$, as was the case for the results presented here for $b=0.7$, or decreases with $\alpha$, which was the case for $b=1$ which was presented in [Bulelzai and Dubbeldam 2012].

\section{Conclusions}

The evolution of atherosclerotic plaque is explored by varying different parameters which affect plaque growth in arteries. We explored codimension-two bifurcation diagrams for a model of atherosclerosis in which the wall shear stress was assumed to be a parameter. We showed that the dynamics is governed by a Bogdanov-Takens point that acts as an organizing center. Depending on the value of $e$, a parameter that controls at what rate oxidized LDL disappears from the plaque, the order of homoclinic and the Hopf curve emanating from the BT-point changes. Crossing of homoclinic and Hopf curves gives rise to a few extra regions in the bifurcation diagram which are so tiny, however, that observing these in experiments will be extremely difficult.

The bifurcation analysis was next used to analyze a self-consistent model in which the shear stress was no longer a parameter, but evolved dynamically. By using techniques from slow-fast systems, we explored the growth of plaques for this more realistic model. Depending on the values of the conversion rate $b$ of the macrophages we find that larger plaques results from higher blood velocities $(b<1)$, or larger plaques correspond to smaller blood velocities $(b \geq 1)$, suggesting that $b>1$ is the relevant parameter regime.

Our analysis reveals that before plaques start to grow they will generally go through a period where the concentrations of the plaque constituents oscillate, before the plaque starts to grow significantly. If this behavior is robust and remains even in more complicated models, this precursor of the onset of artery occlusion could be an indicator that might eventually be used in a clinical environment. Of course, one should realize that the model we investigated in this paper lacks a lot of components that will modify, at least quantitatively 
but possibly also qualitatively, the behavior of the system. For example, the shape of the plaque is entirely neglected and this will lead to changes in the flow profile and the wall shear stress is therefore no longer constant along the artery. Moreover, cytokines and HDL particles have not been taken into account, which have been shown to influence plaque growth [Ougrinovskaia et al. 2010]. The bifurcation study we performed here illustrates its relevance to identiying parameter regions that are clinically sensible and makes predictions about the dynamical behavior of plaques that may be put to a clinical test.

\section{Acknowledgment}

MAKB and JLAD would like to thank Wim van Horssen for scientific discussions. JLAD is grateful to Sebastian Wieczoreck and Bernd Krauskopf for their help with AUTO and for stimulating discussions about the dynamics in the investigated models.

\section{Appendix A}

To calculate the stability of the equilibria of class $I$, we shift the equilibria $\left(0, M_{0}, 0\right)$ to the origin $O$, by introducing $\bar{M}=M-M_{0}$. We may then cast equations (2.1) in the following form

$$
\left(\begin{array}{c}
\dot{m} \\
\dot{\bar{M}} \\
\dot{L}
\end{array}\right)=A\left(\begin{array}{c}
m \\
\bar{M} \\
L
\end{array}\right)+\left(\begin{array}{c}
\frac{a}{1+\sigma} m L+O\left(\|m, \bar{M}, L\|^{3}\right) \\
-b \bar{M} L+b M_{0} L^{2}+O\left(\|m, \bar{M}, L\|^{3}\right) \\
-\frac{d}{f^{2}} m^{2}-e \bar{M} L+O\left(\|m, \bar{M}, L\|^{3}\right)
\end{array}\right)
$$

where we defined the matrix $A$ by

$$
A=\left(\begin{array}{ccc}
-c-\epsilon & 0 & 0 \\
c & 0 & -b M_{0} \\
\frac{d}{f} & 0 & -e M_{0}-1
\end{array}\right)
$$

The eigenvalues of $A$ are easily found to be $\lambda_{1}=-(\epsilon+c), \lambda_{2}=-\left(e M_{0}+1\right), \lambda_{3}=0$. The corresponding eigenvectors are given by $\mathbf{v}_{1}=\left(-\frac{f\left(-e M_{0}-1+\epsilon+c\right)}{d}, \frac{f\left(-e M_{0}-1+\epsilon+c\right) c+d b M_{0}}{d(\epsilon+c)}, 1\right)^{T}, \mathbf{v}_{2}=\left(0, b M_{0} /\left(e M_{0}+1\right), 1\right)^{T}$, and $\mathbf{v}_{3}=(0,1,0)^{T}$.

\subsection{Equilibria of Type II}

Equilibria $I I$ are given as:

$$
\begin{aligned}
& L^{*}=\frac{(\epsilon+c)(1+\sigma)}{a-(1+\sigma)(\epsilon+c)}, \\
& M^{ \pm}=\frac{c m^{*}\left(1+L^{*}\right)}{b L^{*}}, \\
& \left(m^{*}\right)^{2}+m^{*}\left[f-\frac{d b}{e c}+\frac{(1+\sigma)(1+d) b(\epsilon+c)}{e a c}\right]+\frac{f b(1+\sigma)(\epsilon+c)}{e a c}=0 .
\end{aligned}
$$

Solving the quadratic equation for $m^{*}$ gives two values $m^{+}$and $m^{-}$, which satisfy:

$m^{ \pm}=-\frac{f-\frac{d b}{e c}+\frac{(1+\sigma)(1+d) b(\epsilon+c)}{e a c}}{2} \pm \frac{\sqrt{\Delta}}{2}, \quad$ where $\Delta=\left[f-\frac{d b}{e c}+\frac{(1+\sigma)(1+d) b(\epsilon+c)}{e a c}\right]^{2}-4 \frac{b f(1+\sigma)(\epsilon+c)}{e a c}$.

Since the equation is quadratic in $m^{*}$, the two roots have either both positive or both negative. In order for equilibria (II) to exist and being both positive, the following conditions must hold: 


$$
\mathrm{C} 1: \quad a>(1+\sigma)(\epsilon+c) \quad \mathrm{C} 2: \quad f-\frac{d b}{e c}+\frac{(1+\sigma)(1+d) b(\epsilon+c)}{e a c}<0 \quad \mathrm{C} 3: \quad \Delta>0 .
$$

The conditions $\mathrm{C} 2$ and $\mathrm{C} 3$ can be summarized in a condition imposed on $d$

$$
d \geq d_{\text {crit }}:=\frac{f e c a+(1+\sigma)(c+\epsilon) b+2 \sqrt{b(1+\sigma)(c+\epsilon) \text { feca }}}{b(a-(1+\sigma)(c+\epsilon))} .
$$

These equilibria are born from a saddle-node bifurcation as shown in [Bulelzai and Dubbeldam 2012]. Here we describe a generic bifurcation of codimension-two to unfold the dynamics these equilibria govern.

\subsection{Bogdanov-Takens singularity calculations}

The system (2.1) at equilibria type II passes through Bogdanov-Takens singularity. We would like to obtain the normal form by center manifold reduction. On the center manifold, the dynamics is described by the reduced equations $x^{\prime}=y, y^{\prime}=a_{1} x^{2}+b_{1} x y$. We will compute the critical normal form coefficients $a_{1}$ and $b_{1}$ and show that $a_{1}$ is never zero, while $b_{1}$ can vanish. For the computation, we know that the double zero eigen-

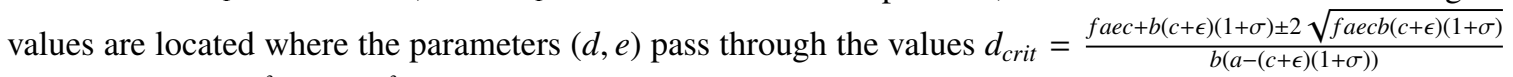
and $e_{c r i t}=\frac{a b^{3}(c+\epsilon)(1+\sigma)^{3}}{f c\left(a^{2}-(c+\epsilon)(1+\sigma)(a+b(1+\sigma))\right)^{2}}$. We chose $e_{c r i t}$ value for the calculation of normal form and not $b_{c r i t}$ as the equation is linear in $e$. The value for $d_{\text {crit }}$ implies a sharp turn (collision of equilibria type II). The value for $m^{ \pm}$at this point is $\frac{\sqrt{f a e c b(1+\sigma)(\epsilon+c)}}{a e c}$. To calculate the critical coefficients, involved in the degeneracy conditions in Bogdanov-Takens bifurcation analysis, we follow [Kuznetsov 1999]. The Jacobian matrix at these critical values, is given by:

$$
J_{c r i t}=\left[\begin{array}{ccc}
0 & 0 & \frac{f((-\epsilon-c) \sigma-c+a-\epsilon)^{2} A}{b(1+\sigma)^{2} a^{2}} \\
c & -\frac{b(\epsilon+c)(1+\sigma)}{a} & -\frac{c f((-1-\sigma) c+(-1-\sigma) \epsilon+a)^{2} A}{a^{2} b(1+\sigma)^{2}(\epsilon+c)} \\
d_{c r i t} f\left(f+\frac{f A}{a b(1+\sigma)}\right)^{-2} & -\frac{e_{c r i t}(\epsilon+c)(1+\sigma)}{a-(1+\sigma)(\epsilon+c)} & \frac{-e_{c r i t} c f A-b^{2}(1+\sigma)^{2}(\epsilon+c)}{b^{2}(1+\sigma)^{2}(\epsilon+c)}
\end{array}\right]
$$

where $A=a^{2}-(\epsilon+c)(1+\sigma)(a+(1+\sigma) b)$. At this bifurcation point, two eigenvalues are zero, and there exist two linearly independent (generalized) eigenvectors $q_{0,1} \in \mathbb{R}^{3}$ for $J_{\text {crit }}$, and two eigenvectors $p_{0,1} \in \mathbb{R}^{3}$ for its transpose such that

$$
J_{\text {crit }} q_{0}=0, \quad J_{\text {crit }} q_{1}=q_{0}, \quad J_{\text {crit }}^{T} p_{1}=0, \quad J_{\text {crit }}^{T} p_{0}=p_{1} .
$$

The eigenvectors are given by:

$$
q_{0}=\left[\begin{array}{c}
\frac{(\epsilon+\sigma \epsilon+c+\sigma c) b}{a c} \\
1 \\
0
\end{array}\right], \quad p_{1}=\left[\begin{array}{c}
\frac{(a-(1+\sigma)(\epsilon+c)) c a^{2}}{-(1+\sigma)^{3}(\epsilon+c)^{2} b^{2}+(1+c+\epsilon)(a-(1+\sigma)(\epsilon+c)) a(1+\sigma) b+(a-(1+\sigma)(\epsilon+c)) a^{2}} \\
-\frac{(1+\sigma)(\epsilon+c)((-\epsilon-c) \sigma-c+a-\epsilon) b a}{-(1+\sigma)^{3}(\epsilon+c)^{2} b^{2}+(1+c+\epsilon)(a-(1+\sigma)(\epsilon+c)) a(1+\sigma) b+(a-(1+\sigma)(\epsilon+c)) a^{2}} \\
\frac{\left(-b(1+\sigma)^{2}(\epsilon+c)+((-\epsilon-c) \sigma-c+a-\epsilon) a\right)^{2}((-\epsilon-c) \sigma-c+a-\epsilon)^{2} f c}{\left(-(1+\sigma)^{3}(\epsilon+c)^{2} b^{2}+(1+c+\epsilon)((-\epsilon-c) \sigma-c+a-\epsilon)(1+\sigma) a b+((-\epsilon-c) \sigma-c+a-\epsilon) a^{2}\right)(1+\sigma)^{2} b a}
\end{array}\right],
$$

and the generalized eigenvectors are given by:

$$
p_{0}=\left[\begin{array}{c}
1 \\
\frac{-(1+\sigma)(\epsilon+c) b+a c}{a c} \\
g_{0}
\end{array}\right], \quad q_{1}=\left[\begin{array}{c}
g_{1} \\
g_{2} \\
\frac{b^{2} a(1+\sigma)^{3}(\epsilon+c)}{f\left(-b(\epsilon+c) \sigma^{2}-(\epsilon+c)(a+2 b) \sigma+(-a-b) \epsilon+(-a-b) c+a^{2}\right)((-\epsilon-c) \sigma-c+a-\epsilon)^{2} c} \\
14
\end{array}\right] .
$$


where the lengthy expressions $g_{0}, g_{1}, g_{2}$ are given by

$$
\begin{aligned}
& g_{0}=\frac{-\left(a^{2}-(1+\sigma)(\epsilon+c) a-b(1+\sigma)^{2}(\epsilon+c)\right)^{2}(a-(1+\sigma)(\epsilon+c)) f}{\left(a^{3}+((1+c+\epsilon) b-\epsilon-c)(1+\sigma) a^{2}-b(1+\sigma)^{2}(1+c+\epsilon)(\epsilon+c) a-(1+\sigma)^{3}(\epsilon+c)^{2} b^{2}\right)(\epsilon+c)(1+\sigma)^{2} b a^{3}} \times \\
& {\left[\left((-1+c) \epsilon+c^{2}\right) a^{3}-(\epsilon+c)(1+\sigma)\left((1+c+\epsilon) b+(-1+c) \epsilon+c^{2}\right) a^{2}\right.} \\
& \left.+b(1+\sigma)^{2}(1+\epsilon)(\epsilon+c)^{2} a+b^{2}(1+\sigma)^{3}(\epsilon+c)^{3}\right] \text {, } \\
& g_{1}=\frac{1}{\left(-(1+\sigma)^{3}(\epsilon+c)^{2} b^{2}+(1+c+\epsilon)(a-(1+\sigma)(\epsilon+c)) a(1+\sigma) b+(a-(1+\sigma)(\epsilon+c)) a^{2}\right)((-1-\sigma) c-\sigma \epsilon+a-\epsilon) c^{2} a^{3}} \times \\
& {\left[-(1+\sigma)^{7}(\epsilon+c)^{5} b^{5}+2\left((1+c+\epsilon) a-\frac{1}{2}(c+2 \epsilon+2)(\epsilon+c)(1+\sigma)\right)(\epsilon+c)^{3}(1+\sigma)^{5} a b^{4}\right.} \\
& -\left((1+c+\epsilon)^{2} a+(\epsilon+c)\left(c^{2}-2 c-\epsilon^{2}-4 \epsilon-1\right)(1+\sigma)\right)(\epsilon+c)(a-(1+\sigma)(\epsilon+c))(1+\sigma)^{3} a^{2} b^{3} \\
& +\left((1+c+\epsilon)\left(c^{2}+(-1+\epsilon) c-2 \epsilon\right) a-(\epsilon+c)\left(c^{3}+(1+2 \epsilon) c^{2}+\left(-1+\epsilon^{2}-\epsilon\right) c-2 \epsilon-2 \epsilon^{2}\right)(1+\sigma)\right) \\
& (a-(1+\sigma)(\epsilon+c))(1+\sigma)^{2} a^{3} b^{2}+\left(c^{2}+(1+\epsilon) c-\epsilon\right)(a-(1+\sigma)(\epsilon+c))^{2}(1+\sigma) a^{4} b+ \\
& \left.(a-(1+\sigma)(\epsilon+c))^{2} c a^{5}\right] \text {, }
\end{aligned}
$$

and

$$
\begin{aligned}
g_{2}= & \frac{1}{\left(-(1+\sigma)^{3}(\epsilon+c)^{2} b^{2}+(1+c+\epsilon)(a-(1+\sigma)(\epsilon+c)) a(1+\sigma) b+(a-(1+\sigma)(\epsilon+c)) a^{2}\right)((-\epsilon-c) \sigma-c+a-\epsilon) c a^{2}} \times \\
& \quad-(1+\sigma)^{6}(\epsilon+c)^{4} b^{4}+2\left((1+c+\epsilon) a-\frac{1}{2}(c+2 \epsilon+2)(\epsilon+c)(1+\sigma)\right)(\epsilon+c)^{2}(1+\sigma)^{4} a b^{3} \\
& \quad-(a-(1+\sigma)(\epsilon+c))\left((1+c+\epsilon)^{2} a+(\epsilon+c)\left(c^{2}-3 c-\epsilon^{2}-4 \epsilon-1\right)(1+\sigma)\right)(1+\sigma)^{2} a^{2} b^{2} \\
& \left.+(1+c+\epsilon)(c-2)(a-(1+\sigma)(\epsilon+c))^{2} a^{3}(1+\sigma) b-(a-(1+\sigma)(\epsilon+c))^{2} a^{4}\right] .
\end{aligned}
$$

The coefficients $a_{1}$ and $b_{1}$ involved in the degeneracy conditions, are given as:

$$
a_{1}=\left\langle p_{1}, B\left(q_{0}, q_{0}\right)\right\rangle \quad \text { and } \quad b_{1}=\left\langle p_{0}, B\left(q_{0}, q_{0}\right)\right\rangle+\left\langle p_{1}, B\left(q_{0}, q_{1}\right)\right\rangle,
$$

where $B(.,$.$) is the multilinear form of the Hessian of (2.1), and for two vectors u$ and $v$ is calculated as :

$B(u, v)=\left[\frac{a\left(u_{1} v_{3}+u_{3} v_{1}\right)}{\left(1+L^{*}\right)^{2}(1+\sigma)}-\frac{2 a m^{+} u_{3} v_{3}}{(1+\sigma)\left(1+L^{*}\right)^{3}},-\frac{b\left(u_{2} v_{3}+u_{3} v_{2}\right)}{\left(1+L^{*}\right)^{2}}+\frac{2 b M^{+} u_{3} v_{3}}{\left(1+L^{*}\right)^{3}},-\frac{2 d f u_{1} v_{1}}{\left(f+m^{+}\right)^{3}}-e\left(u_{2} v_{3}+u_{3} v_{2}\right)\right]$.

Calculating the coefficients at the critical values of parameters, we obtain:

$$
a_{1}=-\frac{(1+\sigma)^{4}(\epsilon+c)^{3} b^{4}}{(a+(1+\sigma) b)\left(-(1+\sigma)^{3}(\epsilon+c)^{2} b^{2}+(1+\sigma) a((-\epsilon-c) \sigma-c-\epsilon+a)(1+c+\epsilon) b+a^{2}((-\epsilon-c) \sigma-c-\epsilon+a)\right) f c},
$$

For all positive parameters, it is evident that the critical normal form coefficient $a_{1}$ is nonzero. The condition $B T .1$ is thus satisfied. The other parameter is calculated as:

$b_{1}=-\frac{\left((1+\sigma)^{3}(\epsilon+c)^{2} b^{2}-(1+\sigma) a(a-(1+\sigma)(\epsilon+c))(1+c+\epsilon) b+a^{2}(a-(1+\sigma)(\epsilon+c))\right)(\epsilon+c)^{2} b^{3}(1+\sigma)^{3}}{f c\left(-(1+\sigma)^{3}(\epsilon+c)^{2} b^{2}+(1+\sigma) a(a-(1+\sigma)(\epsilon+c))(1+c+\epsilon) b+a^{2}(a-(1+\sigma)(\epsilon+c))\right)^{2}}$.

This parameter may change sign, resulting in a (simple) degenerate BT bifurcation. The condition BT.2 is satisfied only except when $b_{1} \neq 0$. So, on this point, there is a codimension-three bifurcation point. This point is a value of $b$ such that:

$$
c=\frac{-2 b^{2} \sigma^{2} \epsilon-a b \sigma-2 a b \sigma \epsilon-4 b^{2} \sigma \epsilon-2 b^{2} \epsilon-2 a b \epsilon+a^{2}+b a^{2}-a b \pm \sqrt{g_{4}}}{2 b(1+\sigma)(a+b+b \sigma)},
$$

where,

$g_{4}=a^{4}-2 a^{3} b \sigma-2 a^{3} b-2 a^{4} b+b^{2} a^{4}+\sigma^{2} b^{2} a^{2}-2 a^{3} b^{2} \sigma+4 b^{3} \sigma^{2} a^{2}+8 a^{2} b^{3} \sigma+2 a^{2} b^{2} \sigma+4 b^{3} a^{2}+b^{2} a^{2}-2 a^{3} b^{2}$.

One of these values is positive. Since, we considered $e_{\text {crit }}$ instead of $b_{\text {crit }}$ for normal form computation, we can easily change them to understand the results in this paper. For the parameters, we chose in the first part of this paper, where $b$ was varied along with $d$, the value of $e$ at which a codimension-three bifurcation occurs is found to be approximately $e=1$.7. Similarly, in the later part of this paper, when we varied $\sigma$ and $d$, the value of $e$ at which a codimension-three bifurcation occurs is $e=1.45$. This value following the above calculations agrees with our Matcont computations [Dhooge et al. 2008]. 


\section{Appendix B}

We calculated the first order approximation for the slow manifold using Eq. (4.4) by solving the following set of equations simultaneously:

$$
P_{1}^{1}=\left[\begin{array}{ccc}
0 & 0 & \frac{a m_{0}^{ \pm}(\sigma)}{(1+\sigma)\left(1+L_{0}^{*}(\sigma)\right)^{2}} \\
c & -\frac{b L_{0}^{*}(\sigma)}{1+L_{0}^{*}(\sigma)} & -\frac{c m_{0}^{ \pm}(\sigma)}{L_{0}^{*}(\sigma)\left(1+L_{0}^{*}(\sigma)\right)} \\
\frac{d f}{\left(f+m_{0}^{ \pm}(\sigma)\right)^{2}} & -e L_{0}^{*}(\sigma) & -\frac{e c m_{0}^{ \pm}(\sigma)(1+L)}{b L_{0}^{*}(\sigma)}-1
\end{array}\right]\left[\begin{array}{c}
m_{1}^{ \pm}(\sigma) \\
M_{1}^{ \pm}(\sigma) \\
L_{1}^{*}(\sigma)
\end{array}\right]=g\left[\begin{array}{c}
\frac{d}{d \sigma} m_{0}^{ \pm}(\sigma) \\
\frac{d}{d \sigma} M_{0}^{ \pm}(\sigma) \\
\frac{d}{d \sigma} L_{0}^{*}(\sigma)
\end{array}\right]
$$

For the second order approximation for slow manifold, the following system was solved simultaneously (coming from Equation (4.4)):

$$
\begin{gathered}
P_{2}^{1}=\left[\begin{array}{ccc}
0 & 0 & \frac{a m_{0}^{ \pm}(\sigma)}{(1+\sigma)\left(1+L_{0}^{*}(\sigma)\right)^{2}} \\
c & -\frac{b L_{0}^{*}(\sigma)}{1+L_{0}^{*}(\sigma)} & -\frac{c m_{0}^{ \pm}(\sigma)}{L_{0}^{*}(\sigma)\left(1+L_{0}^{*}(\sigma)\right)} \\
\frac{d f}{\left(f+m_{0}^{ \pm}(\sigma)\right)^{2}} & -e L_{0}^{*}(\sigma) & -\frac{e c m_{0}^{ \pm}(\sigma)(1+L)}{b L_{0}^{*}(\sigma)}-1
\end{array}\right]\left[\begin{array}{c}
m_{2}^{ \pm}(\sigma) \\
M_{2}^{ \pm}(\sigma) \\
L_{2}^{*}(\sigma)
\end{array}\right]=g\left[\begin{array}{c}
\frac{d}{d \sigma} m_{1}^{ \pm}(\sigma) \\
\frac{d}{d \sigma} M_{1}^{ \pm}(\sigma) \\
\frac{d}{d \sigma} L_{1}^{*}(\sigma)
\end{array}\right] \\
+\left(\left(\begin{array}{c}
\frac{d}{d \sigma} m_{0}^{ \pm}(\sigma) \\
\frac{d}{d \sigma} M_{0}^{ \pm}(\sigma) \\
\frac{d}{d \sigma} L_{0}^{*}(\sigma)
\end{array}\right]\right)\left(\left[\begin{array}{lll}
0 & \left.\frac{\partial g}{\partial M}\right|_{P_{0}^{1}} & \left.\frac{\partial g}{\partial L}\right|_{P_{0}^{1}}
\end{array}\right]\left[\begin{array}{c}
m_{1}^{ \pm}(\sigma) \\
M_{1}^{ \pm}(\sigma) \\
L_{1}^{*}(\sigma)
\end{array}\right]\right)-\frac{1}{2}\left[\begin{array}{ccc}
0 & 0 & \frac{a}{(1+\sigma)\left(1+L_{0}^{*}\right)^{2}} \\
0 & 0 & 0 \\
0 & -e & \frac{e c m_{0}^{ \pm}}{b L_{0}^{* 2}}
\end{array}\right]\left[\begin{array}{c}
m_{1}^{ \pm 2}(\sigma) \\
M_{1}^{ \pm 2}(\sigma) \\
L_{1}^{* 2}(\sigma)
\end{array}\right]
\end{gathered}
$$

\section{References}

[Arnold and Jones 1994] Arnold, L., \& Jones, C., (1994). Dynamical systems: Lecture notes in Mathematics,

[Barra et al.] Barra. J., Armentano, R., Levenson J., Fischer E, Pichel. R., Simon A., (1993) Assessment of smooth muscle cell contribution to descending thoracic aortic elastic mechanics in conscious dogs, Circ. Res 73, 1040-1050.

[Bogdanov 1981] Bogdanov, R.I. (1981) Versal deformations of a singular point on the plane in the case of zero eigenvalues. Sel. Math. Sov. 1, 389-421. (in English)

[Bulelzai and Dubbeldam 2012] Bulelzai, M.A.K., \& Dubbeldam, J.L.A.(2012). Long time evolution of atherosclerotic plaques. J. Theo. Bio., 297, 1-10.

[Bulelzai et al. 2013] Bulelzai M.A.K., Dubbeldam, J.L.A., \& Lahaye D.J.P.,(2013) Atherosclerotic plaque growth by shear stress dependent low-density lipoprotein transfer: the effect of recirculation, Submitted to Journal of Theoretical Biology.

[Crowther 2005] Crowther, M. A. (2005). Pathogenesis of atherosclerosis. ASH Education Program Book, $2005(1), 436-441$.

[Dhooge et al. 2008] Dhooge, A., Govaerts, W., Kuznetsov, Y.A., Meijer, H.G.E. and Sautois, B. (2008). New features of the software MatCont for bifurcation analysis of dynamical systems, Mathematical and Computer Modelling of Dynamical Systems, Vol. 14, No. 2, pp 147-175, 2008.

[Doedel and Oldeman 2012] Doedel E.J. and Oldeman B.E. (2012). AUTO-07P:Continuation and bifurcation software for ordinary differential equations. Available at http://sourceforge.net/projects/auto-07p/

[Dumortier 1993] Dumortier, F. (1993). Techniques in the theory of local bifurcations: Blow-up, normal forms, nilpotent bifurcations, singular perturbations. In Bifurcations and Periodic Orbits of Vector Fields (pp. 19-73). Springer Netherlands.

[Dumortier et al. 1991] Dumortier, F., Roussarie, R. \& Sotomayor, I. (1991) Generic 3-parameter families of planar vector fields. Unfoldings of saddle, focus and elliptic singularities with nilpotent linear parts, Springer Lecture Notes in Mathematics 1480, pp1-164.

[Frisdal et al. 2011] Frisdal E., Lesnik Ph., Olivier M.,Robilard P., Chapman M.J., Huby Th., Guerin M., Le Goff W., (2011) Interleukin6 protects human macrophages from cellular cholesterol accumulation and attenuates the pro-inflammatory response, J. Biol. Chem., M111.264325v1.

[Gear et al 2005] Gear, C. W., Kaper, T. J., Kevrekidis, I. G., \& Zagaris, A. (2005). Projecting to a slow manifold: Singularly perturbed systems and legacy codes. SIAM Journal on Applied Dynamical Systems, 4(3), 711-732.

[Gijsen et al. 2008] Gijsen, F.J.H., Wentzel, J.J., Thury, A., Mastik, F., Schaar, J.A.,Schuurbiers J.C.H., Slager, C.J., van der Giesen, W.J.,de Feyter, P.J., van der Steen, \& A.F.W., Serruys P.W. (2008). Strain distribution in human coronary arteries relates to shear stress, Am. J. Physiol. Heart Circ. Physiol. 295, H1608-H1614.

[Guckenheimer and Holmes 1983] Guckenheimer, J., \& Holmes, P. (1983). Nonlinear oscillations, dynamical systems, and bifurcations of vector fields (Vol. 42). New York: Springer-Verlag.

[Guckenheimer et al. 2012] Guckenheimer, J., Johnson, T., \& Meerkamp, P. (2012). Rigorous Enclosures of a Slow Manifold. SIAM Journal on Applied Dynamical Systems, 11(3), 831-863. 
[Holzapfel et al. 2000] Holzapfel, G.A., Gasser, T.C., \& Ogden, R.W. (2000). A new constitutive framework for arterial wall mechanics and a comparative study of material models, J. Elasticity 61, 1-48. http://www.sens.org/node/1490.

[Kaper et al 2002] Kaper, H. G., \& Kaper, T. J. (2002). Asymptotic analysis of two reduction methods for systems of chemical reactions. Physica D: Nonlinear Phenomena, 165(1), 66-93.

[Krakauer 2007] Krakauer, J.W., (2007). The complex dynamics of stroke onset and progression, Curr Opin Neurol, 20(1):47-50.

[Kokubu et al. 2004] Kokubu, H., Roussarie, R., (2004) Existence of a Singularly Degenerate Heteroclinic Cycle in the Lorenz System and its Dynamical Consequences: Part I, J. of Dyn. and Diff. Eq., Vol. 16, No. 2.

[Kuznetsov 1998] Kuznetsov, Y.A. (1998) Elements of Applied Bifurcation Analysis, Springer Berlin.

[Kuznetsov 1999] Kuznetsov, Y.A. (1999). Numerical normalization techniques for all codim 2 bifurcations of equilibria in ODE's. SIAM journal on numerical analysis, 36(4), 1104-1124.

[Ougrinovskaia et al. 2010] Ougrinovskaia, A., Thompson, R.S., \& Myerscough, M.R., (2010). An ODE model of early stages of atherosclerosis: mechanisms of the inflammatory response, Bull. Math. Biol. 72, 1534-1561 (2010).

[Peng and Jiang 2011] Peng, G., \& Jiang, Y. (2011). Practical computation of normal forms of the Bogdanov-Takens bifurcation. Nonlinear Dynamics, 66(1-2), 99-132.

[Popovic et al. 2004] Popovic, N., Szmolyan, P. (2004) A geometric analysis of the Lagerstrom model problem, J. Differential Equations 199, 290-325.

[Revel et al. 2008] Revel, G., Alonso, D. M., \& Moiola, J. L. (2008). Bifurcation theory applied to the analysis of power systems. Revista de la Unin Matemtica Argentina, 49(1), 1-14.

[Ross 1993] Ross, R. (1993). Atherosclerosis: a defense mechanism gone awry. Am. J. Pathol. 143: 987-1002.

[Shilnikov et al 2001] Shilnikov, L. P., Shilnikov, A. L., Turaev, D. V., \& Chua, L. O. (2001). Methods of qualitative theory in nonlinear dynamics. Singapore: World Scientific.

[Slager et al. 2005] Slager, C. J., Wentzel, J. J., Gijsen, F. J. H., Thury, A., Van der Wal, A. C., Schaar, J. A., \& Serruys, P. W. (2005). The role of shear stress in the destabilization of vulnerable plaques and related therapeutic implications. Nature clinical practice cardiovascular medicine, 2(9), 456-464.

[Wieczorek et al. 2011] Wieczorek, S., Ashwin, P., Luke, C. M., \& Cox, P. M. (2011). Excitability in ramped systems: the compostbomb instability.

[Desroches et al. 2008] Desroches M., Krauskopf B., Osinga H.M., (2008). The geometry of slow manifolds near a folded node, SIAM J. Appl. Dyn. Syst. 7, 1131. Proceedings of the Royal Society A: Mathematical, Physical and Engineering Science, 467(2129), 1243-1269.

[Zohdi et al. 2004] Zohdi, T.J., Holzapfel, G.A. Berger, S.A.,(2004) A phenomenological model for atherosclerotic plaque growth and rupture, J. Theor. Biol. 227, 437-443. 